\title{
PERFIL EPIDEMIOLÓGICO DAS INTERNAÇÕES POR FEBRE HEMORRÁGICA DA DENGUE NO NORDESTE BRASILEIRO
}

\author{
EPIDEMIOLOGICAL PROFILE OF HOSPITALIZATION FOR \\ DENGUE'S HEMORRHAGIC FEVER IN NORTHEAST BRAZIL
}

Isabela Alice Soares de Medeiros ${ }^{1}$

Isabella Rodrigues Destefani ${ }^{2}$

Leandro Januário de Lima $^{3}$

Maria do Carmo Andrade Duarte de Farias ${ }^{4}$

RESUMO: Objetivo: Identificar o perfil epidemiológico das internações por febre hemorrágica devido ao vírus da dengue no Nordeste brasileiro entre 2010 e 2019. Método: Trata-se de um estudo descritivo, retrospectivo, com dados secundários obtidos do Sistema de Informações Hospitalares do Sistema Único de Saúde (SIH/SUS). Foram analisadas as variáveis: número de internações, número de óbitos, unidade da Federação, sexo, cor/raça e faixas etárias. Os dados obtidos foram analisados no software IBM SPSS Statistics 20. Resultados: As internações no Nordeste representaram $34,2 \%$ do total de internações em todo o Brasil $(\mathrm{n}=$ 24.488). O pico de casos se deu em 2010-11, quando se estabeleceu uma tendência de queda até 2017, quando a curva se inverteu e retornou o crescimento. Quanto ao desfecho, 3,64\% morreram durante a internação. Pernambuco teve a maior taxa de prevalência no início da série temporal com Sergipe no outro oposto. Em 2019, o estado de Sergipe tornou-se o estado com a maior taxa de prevalência e o Ceará no extremo oposto. Crianças entre 5 a 9 anos (20,9\%), pacientes de cor parda (39\%) e mulheres (51\%) foram grupos mais prevalentes. Conclusão: A morbidade hospitalar por febre hemorrágica da dengue apresentou um período de queda de oito anos (2010-2017), mas apresenta tendência de crescimento nos últimos anos.

\footnotetext{
1 Graduanda do Curso de Medicina da Universidade Federal de Campina Grande. E-mail: isabelaalicesm@gmail.com.

${ }_{2}$ Graduanda do Curso de Medicina da Universidade Federal de Campina Grande. E-mail: isadestefani97@gmail.com.

3 Graduando do Curso de Medicina da Universidade Federal de Campina Grande. E-mail: leandrojanuario100@gmail.com.

4 Professora Titular. Doutora em Enfermagem, Universidade Federal de Campina Grande (UFCG/CFP/UACV). E-mail: carmofarias0@gmail.com.
} 
Palavras chave: Dengue. Dengue Grave. Epidemiologia. Hospitalização. Perfil de Saúde.

ABSTRACT: Objective: To identify the epidemiological profile of hospitalizations for hemorrhagic fever due to the dengue virus in Northeast Brazil between the 2010 and 2019 period. Method: This is a descriptive, retrospective study, with secondary data obtained from the Hospital Information System of the Unified Health System (SIH/SUS). The variables analyzed were number of hospitalizations, number of deaths, Federation unity, sex, color/race, and age groups. The data obtained was analyzed using the IBM SPSS Statistics 20 software. Results: Hospitalizations in the Northeast represented $34.2 \%$ of the total hospitalizations throughout Brazil ( $n=$ 24,488). The peak of cases occurred in 2010-11 next a downward trend was established, until 2017, where the curve was reversed, and the growth returned. Regarding the outcome, 3.64\% died during hospitalization. Pernambuco had the highest prevalence rate at the beginning of the time series with Sergipe in the opposite side. In 2019, the state of Sergipe became the state with the highest prevalence rate and Ceará in the opposite extreme. Children between 5 and 9 years old (20.9\%), brown patients (39\%) and women (51\%) were the most prevalent groups. Conclusion: Hospital morbidity due to dengue hemorrhagic fever presented a fall period of eight years (2010-2017) but has shown a growth trend in recent years.

Keywords: Dengue. Severe Dengue. Epidemiology. Hospitalization. Health Profile. 


\section{INTRODUÇÃO}

A dengue é uma doença infecciosa causada por um vírus da família Flaviviridae que contém quatro sorotipos, sendo eles DENV-1, DENV-2, DENV-3 e DENV-4. O vírus é transmitido por picadas de fêmeas do mosquito de gênero Aedes, principalmente o Aedes aegypti (PUSTIGLIONE, 2016). Atualmente, é a mais expressiva arbovirose humana, chegando a causar mais de 390 milhões de infecções no mundo todo, com 96 milhões de manifestações clínicas (OPAS, 2019). No Brasil, em 2019, a partir do monitoramento dos casos de arboviroses urbanas transmitidas pelo Aedes, foram notificados mais de um milhão e quinhentos mil casos prováveis (taxa de incidência de 735,2 casos por 100 mil habitantes), sendo a Região Nordeste correspondente à terceira maior taxa de incidência $(376,7$ casos/100 mil habitantes) (BRASIL, 2020).

Após a picada do mosquito, o vírus passa por um período de incubação médio de 4 a 7 dias, podendo ser uma fase sintomática ou assintomática. Os sintomas se caracterizam por febre alta, cefaleia, dor retrorbital, mialgia, artralgia, entre outros, que duram, em média, três dias. Depois desse período, o paciente pode evoluir para um quadro resolutivo ou ter um quadro de febre hemorrágica da dengue (SINGHI; KISSOON; BANSAL, 2007).

A febre hemorrágica da dengue decorre da perturbação do endotélio vascular pelo vírus e pela grande liberação de mediadores inflamatórios, que alteram a permeabilidade vascular, causando extravasamento de plasma sanguíneo, comprovado pela elevação do hematócrito. A perda de plasma intravascular também pode causar hipotensão considerável, derrame pleural e/ou derrame peritoneal (ROTHMAN, 2004). Além disso, a trombocitopenia, contagem de plaquetas abaixo de 100.000 células $/ \mathrm{mm}^{3}$, é um importante achado clínico da doença, responsável por caracterizá-la, que pode levar o paciente a ter hemorragias. Outros sintomas da febre hemorrágica da dengue incluem dor abdominal, hepatomegalia e vômitos (TERCERO; FERNÁNDEZ, 2019). 
O risco de o paciente apresentar dengue hemorrágica é maior quando a segunda infecção é causada pelo sorotipo DENV-2. A evolução de dengue clássica para dengue hemorrágica ocorre em pequena porcentagem de indivíduos, entre 0,3\% a 4\% (FERREIRA, 2012). Esse severo quadro clínico evolui de forma rápida e dura, em média, de 24 a 48 horas (TERCERO; FERNÁNDEZ, 2019).

A febre hemorrágica da dengue vem trazendo grandes desafios para a saúde pública do Brasil. O impacto de um problema de saúde pode ser medido por sua gravidade e pelo valor social que representa para sociedade, isto é, pelo seu impacto real ou potencial e sua repercussão no desenvolvimento socioeconômico (CAVALCANTI et al., 2010). Nesse contexto, quando se considera a prevalência de internações por dengue, em média todos os anos, cerca de 500.000 casos de dengue hemorrágica necessitam desta intervenção. Pelo menos $2,5 \%$ dos casos vão a óbito, mas essa taxa pode ser maior, podendo superar $20 \%$ quando os pacientes não recebem tratamento adequado (SINGHI; KISSOON; BANSAL, 2007).

As internações hospitalares como fonte de dados sobre morbidade têm sido objeto de investigação de diversos estudos (BITTENCOURT; CAMACHO; LEAL, 2006) e se configura como um dos principais instrumentos para avaliação para fins epidemiológicos, pois reúne, a partir das autorizações de internação hospital (AlHs), diversas informações sobre a morbidade e sobre o paciente admitido no hospital, sendo capaz de gerar informação em saúde (LESSA et al., 2000).

Assim, este estudo tem por objetivo identificar o perfil epidemiológico das internações por febre hemorrágica devida ao vírus da dengue na região do Nordeste brasileiro. A escolha por esta região deu-se em virtude de suas particularidades sociodemográficas, econômicas, sanitárias e culturais, que influenciam a estrutura epidemiológica e a distribuição das doenças.

\section{MÉTODO}

Trata-se de um estudo descritivo, transversal e retrospectivo com foco em dados secundários obtidos do Sistema de Informações Hospitalares do Sistema 
Único de Saúde (SIH/SUS). Essa plataforma tem origem nas Autorizações de Internações Hospitalares (AlH) e fornece a possibilidade de prover informações diagnóstica, demográfica e geográfica para cada internação hospitalar, ampliando a possibilidade de produção de conhecimento no campo da Saúde Coletiva (VIACAVA, 2002).

A coleta de dados foi realizada em julho de 2020. Foram analisados os dados entre janeiro de 2010 a dezembro de 2019, que teve como população de estudo todos os casos de internação por "Febre hemorrágica devida ao vírus da dengue", segundo a Lista de Morbidade CID-10, durante o período analisado, na região Nordeste (18,2\% do território nacional) que possui cerca de 50 milhões de habitantes e compreende os estados do Maranhão (MA), Piauí (PI), Ceará (CE), Rio Grande do Norte (RN), Paraíba (PB), Pernambuco (PE), Alagoas (AL), Sergipe (SE) e Bahia (BA) (CÂMARA et al., 2007).

As variáveis investigadas foram número de internações, número de óbitos e, com o fim de traçar o perfil epidemiológico das internações por febre hemorrágica devida ao vírus da dengue, foram analisadas variáveis sociodemográficas: unidade da Federação, sexo, cor/raça e faixas etárias. Os dados obtidos foram organizados e analisados por meio do software IBM SPSS Statistics 20 por meio de estatísticas descritivas.

Neste estudo, não foi necessária apreciação do Comitê de Ética em Pesquisa (CEP) por se tratar de pesquisa em dados secundários, de domínio público, e por não haver relação direta com seres humanos, mas apenas com dados disponíveis no SIH.

\section{RESULTADOS E DISCUSSÕES}

Entre janeiro de 2010 e dezembro de 2019, foram registradas 24.488 internações por febre hemorrágica devida ao vírus da dengue no Brasil. Dentre a distribuição dos números entre as regiões do país, a região Nordeste foi detentora do maior percentual, com 34\%, que corresponde a 8.377 internações. 
Esse domínio pode estar associado ao constatar a região como mais pobre do país, sendo que, diante do cenário de baixa cobertura do saneamento básico e de distribuição de água potável, é comum que as residências possuam reservatórios para armazenamento de água. Eles são fontes potenciais de focos de desova do mosquito (TEIXEIRA; BARRETO; GUERRA, 1999). O Nordeste também apresenta cidades atratoras, com grande potencial turístico, que favorecem a disseminação do vírus e do vetor para o país. Estes espaços atraem cidadãos, seja pelo trabalho ou turismo, que aí se expõem à infecção, tornando uma via de disseminação (CÂMERA et al., 2007). Além disso, estudos comprovaram a influência de fatores climáticos em cidades do Nordeste como ideias para manifestação do Aedes aegypti e aumento da incidência da doença (BARACHO et al., 2014). Temperaturas, níveis adequados de umidade e chuvas fornecem as condições para que o mosquito da dengue se reproduza e transmita a doença (PAREDA; ALVES; RANGEL, 2011).

No Brasil, a primeira epidemia documentada clínica e laboratorialmente foi em 1981-1982 e, desde então, a incidência de dengue é sustentada, intercalando-se com a ocorrência de epidemias, geralmente associadas com a introdução de novos sorotipos (NUNES, 2016). 
Gráfico 1 - Distribuição do número de internações na região Nordeste por febre hemorrágica devida ao vírus da dengue, 2010-2019.

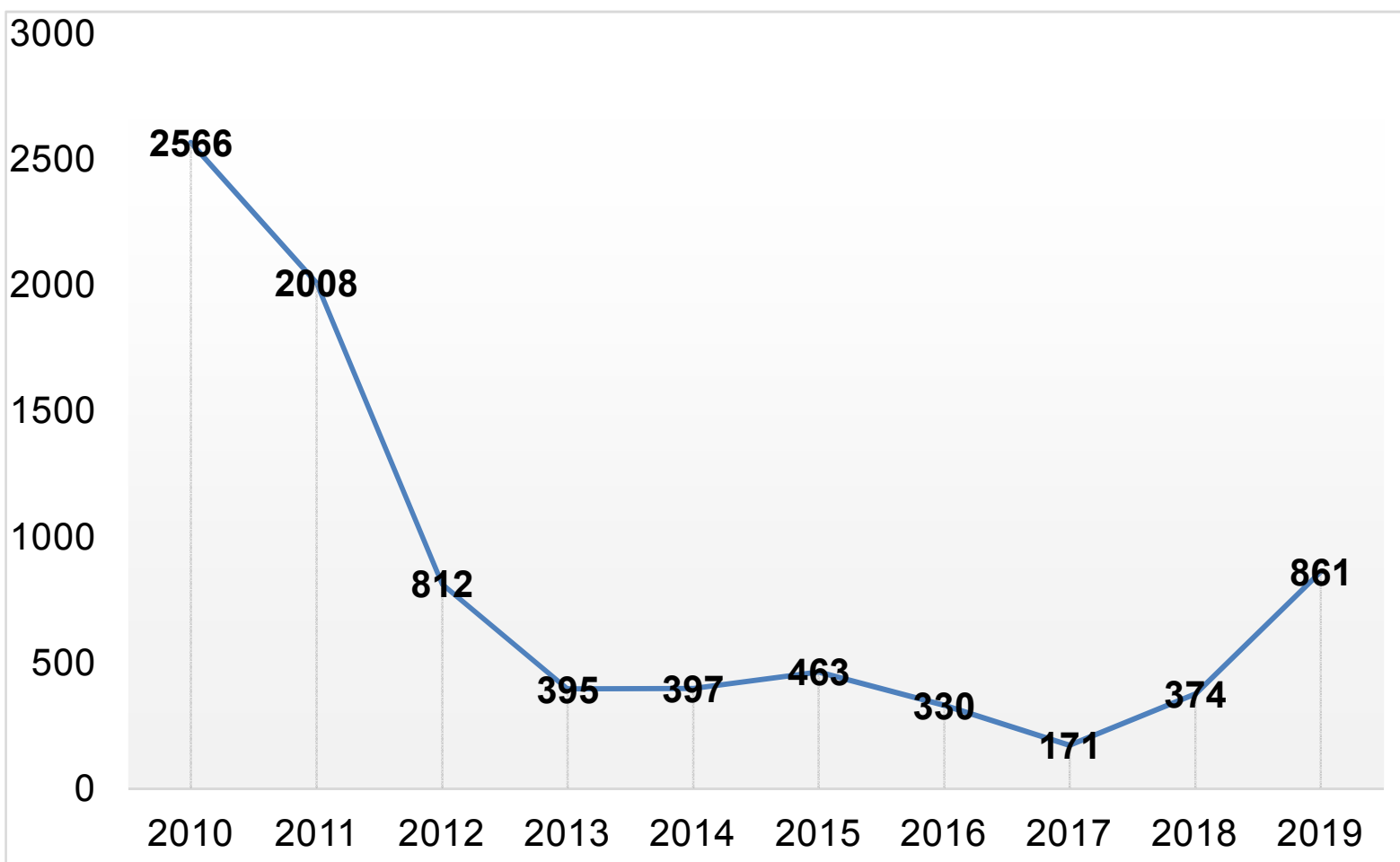

Fonte: Ministério da Saúde - Sistema de Informações Hospitalares do SUS (SIH/SUS).

Diante dos números, exclusivamente na Região Nordeste, no período analisado, verificou-se que o ano com maior prevalência foi 2010 (30,6\%), seguido pelo ano de 2011 (24\%), conforme o gráfico 1. Esse número alto de internações está associado aos vários surtos de dengue que foram documentados em muitas regiões do Brasil, no início de 2010 com a cocirculação de três sorotipos: DENV 1, 2 e 3 (ROMANO et al., 2010).

A tendência de queda teve seu valor inferior em 2017, com apenas $2 \%$ do número total de internações em toda a região Nordeste. Contudo, em seguida, a curva inverte-se com novo crescimento das internações. A redução das internações que dominou o período pode estar associada às melhores e maiores campanhas de conscientização populacional, além da implantação da Estratégia Global para 
Prevenção e do Controle da Dengue pela Organização Mundial da Saúde (CORREIA et al., 2019).

Gráfico 2 - Distribuição dos óbitos hospitalares por febre hemorrágica devida ao vírus da dengue, no Nordeste, por ano de processamento, 2010 e 2019.

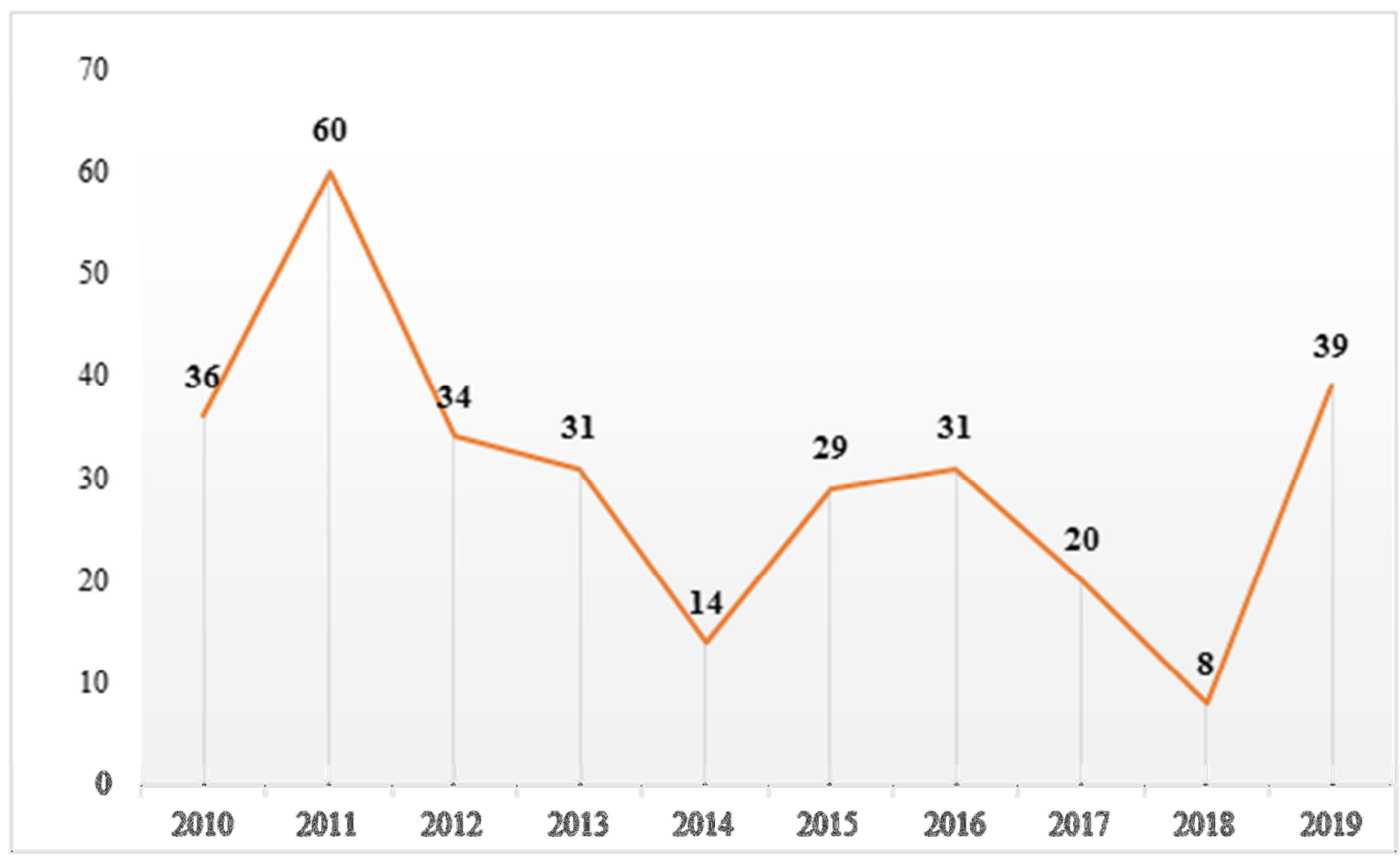

Fonte: Ministério da Saúde - Sistema de Informações Hospitalares do SUS (SIH/SUS).

Um aumento importante na incidência de febre hemorrágica da dengue no Brasil acompanhou a introdução do sorotipo DENV-3. A falta de diagnóstico precoce e de tratamento adequado foi responsável por vários casos fatais de febre hemorrágica da dengue, bastante alta no Brasil, geralmente superiores a $5,5 \%$ (TEIXEIRA et al., 2005).

A circulação sequencial de mais de um sorotipo vem propiciando o aumento na incidência de febre hemorrágica da dengue, com consequente incremento na mortalidade por esta doença (BARRETO; CARMO, 2007). A taxa de mortalidade hospitalar foi de $3,64 \%$ no período estudado, considerando que no período analisado 
o número de óbitos somou um total de 305 casos (Gráfico 2), dentre as 8.377 internações registradas.

Tabela 1 - Internações por febre hemorrágica devida ao vírus da dengue por Unidade da Federação (Região Nordeste) e ano de processamento, 2010-2019.

\begin{tabular}{|c|c|c|c|c|c|c|c|c|c|c|c|c|}
\hline Estado & & 2010 & 2011 & 2012 & 2013 & 2014 & 2015 & 2016 & 2017 & 2018 & 2019 & Total (\%) \\
\hline Maranhão & & 181 & 142 & 40 & 16 & 27 & 54 & 29 & 12 & 7 & 56 & $564(6,7)$ \\
\hline Piauí & & 31 & 24 & 14 & 4 & 8 & 12 & 2 & 2 & 6 & 74 & $177(2,1)$ \\
\hline Ceará & & 141 & 296 & 113 & 86 & 99 & 192 & 61 & 76 & 23 & 60 & $1147(13,7)$ \\
\hline $\begin{array}{l}\text { Rio Grande } \\
\text { Norte }\end{array}$ & do & 196 & 459 & 128 & 55 & 54 & 12 & 40 & 10 & 125 & 49 & $1128(13,5)$ \\
\hline Paraíba & & 130 & 161 & 86 & 63 & 30 & 20 & 40 & 12 & 73 & 100 & $715(8,5)$ \\
\hline Pernambuco & & 978 & 664 & 147 & 31 & 55 & 58 & 59 & 41 & 113 & 176 & $2322(27,7)$ \\
\hline Alagoas & & 138 & 44 & 87 & 12 & 40 & 21 & 10 & 2 & 1 & 40 & $395(4,7)$ \\
\hline Sergipe & & 10 & 28 & 27 & 10 & 18 & 13 & 9 & 2 & 2 & 66 & $185(2,2)$ \\
\hline Bahia & & 761 & 190 & 170 & 118 & 66 & 81 & 80 & 14 & 24 & 240 & $1744(20,8)$ \\
\hline Total & & 2566 & 2008 & 812 & 395 & 397 & 463 & 330 & 171 & 374 & 861 & $8377(100)$ \\
\hline
\end{tabular}

Fonte: Ministério da Saúde - Sistema de Informações Hospitalares do SUS (SIH/SUS).

Ao analisar a distribuição geográfica das internações entre as Unidades da Federação do Nordeste, o Piauí foi o estado que acumulou menor número ( $\mathrm{n}=177$; $2,1 \%)$, seguido por Sergipe $(2,2 \%)$ e Alagoas (4,7\%). No outro extremo, Pernambuco ( $n=2322 ; 27,7 \%)$, Bahia $(20,8 \%)$ e Ceará $(13,7 \%)$ lideraram a prevalência regional.

Essa diferença de distribuição pode ser explicada devido ao fato de certas condições ambientais no Brasil favorecerem a proliferação da patologia, como a temperatura, a pluviometria e a umidade relativa do ar, possibilitando a dispersão do vetor (BARACHO et al., 2014). Diante disso, parte do estado do Piauí, por exemplo, de acordo com o Instituto Nacional de Meteorologia (INMET), possui piores condições climáticas para o desenvolvimento do mosquito e incidência da dengue. Esse fator estende-se para as outras unidades federativas, visto que as variáveis climáticas desempenham um papel importante, com impacto estatisticamente significativo para explicar os casos de dengue (PEREDA; ALVES; RANGEL, 2011). 
Tabela 2 - Taxas de prevalência da febre hemorrágica devida ao vírus da dengue por 100.000 habitantes, Nordeste, 2010-2019.

\begin{tabular}{|c|c|c|c|c|c|c|c|c|c|c|c|c|}
\hline Estado & 2010 & 2011 & 2012 & 2013 & 2014 & 2015 & 2016 & 2017 & 2018 & 2019 & $\bar{x}$ & $\sigma / \bar{x}$ \\
\hline Maranhão & 2.69 & 2.10 & 0.58 & 0.23 & 0.39 & 0.78 & 0.41 & 0.17 & 0.09 & 0.79 & 0,82 & 1,06 \\
\hline Piauí & 0.97 & 0.74 & 0.43 & 0.12 & 0.24 & 0.37 & 0.06 & 0.06 & 0.18 & 2.26 & 0,54 & 1,24 \\
\hline Ceará & 1.63 & 3.40 & 1.29 & 0.97 & 1.11 & 2.15 & 0.68 & 0.84 & 0.25 & 0.65 & 1,29 & 0,70 \\
\hline $\begin{array}{l}\text { Rio Grande } \\
\text { do Norte }\end{array}$ & 6.04 & 14.03 & 3.87 & 1.65 & 1.60 & 0.35 & 1.16 & 0.28 & 3.59 & 1.39 & 3,39 & 1,22 \\
\hline Paraíba & 3.38 & 4.17 & 2.21 & 1.61 & 0.76 & 0.50 & 1.01 & 0.30 & 1.82 & 2.48 & 1,82 & 0,69 \\
\hline Pernambuco & 10.86 & 7.32 & 1.61 & 0.33 & 0.59 & 0.62 & 0.62 & 0.43 & 1.18 & 1.84 & 2,54 & 1,40 \\
\hline Alagoas & 4.31 & 1.36 & 2.69 & 0.36 & 1.22 & 0.64 & 0.30 & 0.06 & 0.03 & 1.19 & 1,21 & 1,11 \\
\hline Sergipe & 0.47 & 1.31 & 1.25 & 0.46 & 0.82 & 0.58 & 0.40 & 0.08 & 0.08 & 2.87 & 0,83 & 0,99 \\
\hline Bahia & 5.32 & 1.32 & 1.17 & 0.81 & 0.45 & 0.55 & 0.54 & 0.09 & 0.16 & 1.61 & 1,20 & 1,26 \\
\hline
\end{tabular}

O Rio Grande do Norte apresentou a maior prevalência média entre todos os estados e o Maranhão apresentou a menor média $(0,82)$, no período total em análise. Pernambuco teve uma distribuição menos homogênea $(C V=1,40)$, ao passo que a Paraíba apresentou a mais homogênea (Tabela 2). Pernambuco também teve a maior taxa de prevalência no início da série temporal com Sergipe no outro oposto. Já em 2019, o estado de Sergipe tornou-se o estado com a maior taxa de prevalência $(2,87)$ e o estado do Ceará como o de menor taxa $(0,65)$. 
Tabela 3 - Internações por febre hemorrágica devida ao vírus da dengue por características sociodemográficas, no Nordeste, 2010-2019.

\begin{tabular}{|c|c|c|c|c|c|c|c|c|c|c|c|}
\hline \multirow{2}{*}{ Variável } & \multicolumn{10}{|c|}{ Ano de processamento } & \multirow{2}{*}{ TOTAL (\%) } \\
\hline & 2010 & 2011 & 2012 & 2013 & 2014 & 2015 & 2016 & 2017 & 2018 & 2019 & \\
\hline TOTAL & 2566 & 2008 & 812 & 395 & 397 & 463 & 330 & 171 & 374 & 861 & $8377(100)$ \\
\hline \multicolumn{12}{|l|}{ Faixa Etária } \\
\hline-1 ano & 107 & 83 & 13 & 5 & 12 & 18 & 16 & 4 & 12 & 22 & $292(3,5)$ \\
\hline 1 a 4 anos & 176 & 182 & 68 & 24 & 24 & 16 & 18 & 15 & 40 & 37 & $600(7,2)$ \\
\hline 5 a 9 anos & 715 & 465 & 119 & 41 & 44 & 49 & 21 & 19 & 72 & 206 & $1751(20,9)$ \\
\hline 10 a 14 anos & 521 & 373 & 130 & 50 & 51 & 53 & 41 & 17 & 68 & 135 & $1439(17,2)$ \\
\hline 15 a 19 anos & 158 & 144 & 55 & 40 & 47 & 49 & 29 & 19 & 48 & 89 & $678(8,1)$ \\
\hline 20 a 29 anos & 315 & 218 & 135 & 76 & 69 & 76 & 43 & 20 & 44 & 129 & $1125(13,4)$ \\
\hline 30 a 39 anos & 204 & 183 & 91 & 40 & 62 & 68 & 25 & 21 & 33 & 83 & $810(9,7)$ \\
\hline 40 a 49 anos & 161 & 149 & 88 & 46 & 39 & 38 & 24 & 14 & 16 & 57 & $632(7,5)$ \\
\hline 50 a 59 anos & 107 & 104 & 53 & 32 & 17 & 45 & 38 & 15 & 18 & 44 & $473(5,6)$ \\
\hline 60 a 69 anos & 61 & 62 & 34 & 18 & 18 & 25 & 28 & 10 & 17 & 25 & $298(3,6)$ \\
\hline 70 a 79 anos & 30 & 29 & 18 & 17 & 10 & 13 & 20 & 11 & 3 & 25 & $176(2,1)$ \\
\hline $\begin{array}{l}80 \text { anos e + } \\
\text { Sexo }\end{array}$ & 11 & 16 & 8 & 6 & 4 & 13 & 27 & 6 & 3 & 9 & $103(1,2)$ \\
\hline Masculino & 1262 & 1026 & 362 & 178 & 190 & 211 & 176 & 81 & 204 & 411 & $4101(49)$ \\
\hline $\begin{array}{l}\text { Feminino } \\
\text { Cor/raça }\end{array}$ & 1304 & 982 & 450 & 217 & 207 & 252 & 154 & 90 & 170 & 450 & $4276(51)$ \\
\hline Branca & 162 & 179 & 63 & 24 & 31 & 19 & 35 & 10 & 59 & 56 & $638(7,6)$ \\
\hline Preta & 34 & 30 & 3 & 3 & 2 & 3 & 2 & 1 & 6 & 25 & $109(1,3)$ \\
\hline Parda & 647 & 885 & 283 & 170 & 172 & 219 & 155 & 92 & 193 & 450 & $3266(39,0)$ \\
\hline Amarela & 6 & 9 & 1 & 3 & 4 & 5 & 9 & 8 & 20 & 63 & $128(1,5)$ \\
\hline Indígena & - & - & - & - & - & - & - & - & - & 1 & $1(0,0)$ \\
\hline $\begin{array}{l}\text { Sem } \\
\text { informação }\end{array}$ & 1717 & 905 & 462 & 195 & 188 & 217 & 129 & 60 & 96 & 266 & $4235(50,6)$ \\
\hline
\end{tabular}

Fonte: Ministério da Saúde - Sistema de Informações Hospitalares do SUS (SIH/SUS).

Ao analisar a distribuição por faixa etária, constata-se que o menor número de internações foi de pacientes com 80 ou mais anos $(n=103 ; 1,2 \%)$ e entre 70 a 79 anos $(2,1 \%)$. O maior número de internações concentrou-se entre as crianças, na faixa etária de 5 a 9 anos $(n=1751 ; 20,9 \%)$ e 10 a 14 anos $(17,2 \%)$.

Ao considerar pacientes pediátricos com a faixa de idade entre 0 e 19 anos, adultos entre 20 e 59 anos e idosos com 60 anos ou mais, verifica-se que as crianças acumulam $56,9 \%$ do total de internações por febre hemorrágica, na região Nordeste, devida ao vírus da dengue, tornando-se o grupo mais acometido à 
complicação, de acordo com os dados. Os adultos somaram $36,2 \%$ e, em minoria, estão os idosos, acumulando $6,9 \%$ do total.

Entretanto, a taxa de letalidade por dengue foi maior entre os idosos a partir dos 60 anos, e, dentro dessa categoria, os mais afetados foram aqueles com 80 anos ou mais. O risco relativo (RR) de morrer por dengue na faixa etária de 80 anos ou mais foi 129,7 vezes maior que na faixa etária de 1 a 4 anos (BRASIL, 2020).

A importância de entender o perfil das pessoas mais propensas a adquirir a morbidade é tática importante para conhecer uma trajetória e assim, intervir. Devese levar em consideração que em uma internação o afastamento do paciente do seu meio de trabalho, a morbimortalidade, entre outros fatores, gera custos para o Sistema Único de Saúde. Portanto, especificar quais os indivíduos mais prováveis de adoecerem possibilita uma intervenção capaz de economizar custos (SHEPARD et al., 2011).

A distribuição de casos entre os sexos apresentou baixo coeficiente de variação $(0,029)$. Tal fato pode ser confirmado ao se observar a distribuição do número entre o sexo feminino e o sexo masculino das internações pelo período estudado. As internações acumuladas pelo sexo masculino corresponderam a $49 \%$ ( $n=4101$ ), revelando uma grande similaridade e constância de número entre os sexos. Tal diferença numérica, em que a incidência é um pouco maior em mulheres, pode ser fundamentada ao considerar que as mulheres buscam mais atendimentos nos serviços de saúde em relação aos homens (AZEVEDO et al., 2013).

Já em relação à cor/raça dos pacientes, apresenta-se o maior coeficiente de variação $(1,33)$ dentre as variáveis sociodemográficas, tendo a maioria das admissões de internação caracterizadas pela falta de informação sobre cor/raça do paciente $(50,6 \%)$, dificultando o delineamento de um perfil preciso em relação à cor/raça, já que os dados faltosos poderiam alterar o panorama do perfil epidemiológico.

No entanto, dentre as informações declaradas $(49,4 \%)$, o maior grupo foi o de cor parda com 39\% e, logo em seguida, a cor branca (7,6\%). Indígenas representaram porcentagem insignificativa $(<1 \%)$, a qual pode ser justificada pela dificuldade de acesso da população indígena ao sistema de saúde brasileiro (PERES; MOURA; AGUIAR, 2020). O vírus da dengue tem suscetibilidade universal 
e a pesquisa de cor/raça torna-se relevante no aspecto social, ao constatar que há um predomínio de pardos em classes mais baixas da sociedade brasileira (CORREIA et al., 2019).

\section{CONCLUSÃO}

A morbidade hospitalar por febre hemorrágica do vírus da dengue no Nordeste apresentou uma tendência de queda constante em uma década. Contudo, os últimos anos apresentam uma tendência à retomada de um pico epidêmico, devendo ser administrada e controlada, com o fim de evitar um novo surto epidêmico.

Pacientes em idade infantil e parda foram mais afetados, o que pode apontar uma tendência de risco para estes grupos na população. Conhecer estas relações de prevalência proporciona condições para a elaboração de planos de contenção e promoção de saúde, com o fim de contribuir para a redução da prevalência hospitalar. 


\section{REFERÊNCIAS BIBLIOGRÁFICAS}

AZEVEDO, A. L. S. et al. Doenças crônicas e qualidade de vida na atenção primária à saúde. Cadernos de saúde pública, [S. I.], v. 29, p. 1774-1782, 2013.

BARACHO, R. C. M. et al. A influência climática na proliferação da dengue na cidade de Areia, Paraíba. Revista Gaia Scientia, [S. I.], v. 8, n. 1, p. 65-73, 2014.

BARRETO, M. L.; CARMO, E. H. Padrões de adoecimento e de morte da população brasileira: os renovados desafios para o Sistema Único de Saúde. Ciência \& Saúde Coletiva, [S. I.], v. 12, p. 1179-1790, 2007.

BITTENCOURT, S. A.; CAMACHO, L. A. B.; LEAL, M. C. O Sistema de Informação Hospitalar e sua aplicação na saúde coletiva. Cadernos de Saúde Pública, [S. I.], v. 22, p. 19-30, 2006.

BRASIL. Ministério da Saúde. Secretaria de Vigilância em Saúde. Boletim Epidemiológico: monitoramento dos casos de arboviroses urbanas transmitidas pelo Aedes Aegypt (dengue, chikungunya e zika), Semanas Epidemiológicas 1 a 52, 2020. v. 51, n. 2, jan. 2020.

CÂMARA, F. P. et al. Estudo retrospectivo (histórico) da dengue no Brasil: características regionais e dinâmicas. Revista da Sociedade Brasileira de Medicina Tropical, [S. I.], v. 40, n. 2, p. 192-196, 2007.

CAVALCANTI, L. P. G. et al. Clinical and epidemiological characterization of dengue hemorrhagic fever cases in northeastern, Brazil. Revista da Sociedade Brasileira de Medicina Tropical, [S. I.], v. 43, n. 4, p. 355-358, 2010.

CORREIA, T. C. et al. Prevalência de dengue clássica e dengue hemorrágica no Brasil, entre 2011 e 2015. Revista Eletrônica Acervo Saúde, [S. I.], n. 22, p. e753-e753, 2019.

FERREIRA, G. L. C. Global dengue epidemiology trends. Rev. Inst. Med. trop. S. Paulo, São Paulo, v. 54, supl. 18, p. 5-6, Oct. 2012.

LESSA, F. J. D. et al. Novas metodologias para vigilância epidemiológica: uso do Sistema de Informações Hospitalares-SIH/SUS. Informe Epidemiológico do SUS, [S. I.], v. 9, p. 3-19, 2000.

NUNES, L, S. Experiência de Maca, /RJ com homeopatia e dengue, 2007-2012. Rev. Homeopatia, São Paulo, v. 79, n. 1/2, p. 1-16, 2016.

OPAS. Organização Pan-americana de Saúde. Indicadores de saúde. Elementos conceituais e práticos. Pan American Health Organization/World Health Organization, Washigton D. C.; 2019.

PEREDA, P. C.; ALVES, D. C. O.; RANGEL, M. A. Elementos climáticos e incidência de dengue: teoria e evidência para municípios brasileiros. In: $33^{\circ}$ Meeting of the Brazilian Econometric Society. 2011.

PERES, A. O.; MOURA, F. M.; AGUIAR, D. M. Saúde Indígena e dificuldades no acesso ao sistema público de saúde no Amazonas. BIUS-Boletim Informativo Unimotrisaúde em Sociogerontologia, [S. I.], v. 19, n. 13, p. 1-11, 2020.

PUSTIGLIONE, M. Medicina do Trabalho e doenças emergentes, reemergentes e negligenciadas: a conduta nos casos da febre da dengue, do Chikungunya e do Zika vírus. Revista Brasileira de Medicina Tropical, Uberaba, v. 14, n. 1, p. 1-12, jan.-abr. 2016. 
ROMANO, C. M. et al. Characterization of Dengue virus type 2: new insights on the 2010 Brazilian epidemic. PloS one, [S. I.], v. 5, n. 7, p. e11811, 2010.

ROTHMAN, A. L. Dengue: defining protective versus pathologic immunity. The Journal of Clinical Investigation, Ann Arbor, [S. I.], v. 113, n. 4, abr. 2004.

SHEPARD, D. S. et al. Economic impact of dengue illness in the Americas. The American journal of tropical medicine and hygiene, [S. I.], v. 84, n. 2, p. 200-207, 2011.

SINGHI, S.; KISSOON, N.; BANSAL, A. Dengue e dengue hemorrágico: aspectos do manejo na unidade de terapia intensiva. Jornal de Pediatria, [S. I.], v. 83, n. 2, p. 22-35, maio, 2007.

TEIXEIRA, M. G. et al. Dengue and dengue hemorrhagic fever epidemics in Brazil: what research is needed based on trends, surveillance, and control experiences?. Cadernos de Saúde Pública, [S. I.], v. 21, n. 5, p. 1307-1315, 2005.

TEIXEIRA, M. G.; BARRETO, M. L.; GUERRA, Z. Epidemiologia e medidas de prevenção do dengue. Informe epidemiológico do SUS, [S. I.], v. 8, n. 4, p. 5-33, 1999.

TERCERO, A. A.; FERNÁNDEZ, R. V. Dengue: presentácion e importancia de fator activación de plaquetas en la evolución de la fase crítica. Revista Médica Sinergia, Guácimo, v. 4, n. 11, nov. 2019.

VIACAVA, F. Informações em saúde: a importância dos inquéritos populacionais. Ciência \& Saúde Coletiva, [S. I.], v. 7, p. 607-621, 2002. 